\title{
Vorticity and particle polarization in heavy ion collisions (exper- imental perspective)
}

\author{
Sergei $A$. Voloshin ${ }^{1, \star}$ \\ ${ }^{1}$ Wayne State University, 666 W. Hancock, Detroit 48201, Michigan, U.S.A.
}

\begin{abstract}
The recent measurements of the global polarization and vector meson spin alignment along the system orbital momentum in heavy ion collisions are briefly reviewed. A possible connection between the global polarization and the chiral anomalous effects is discussed along with possible experimental checks. Future directions, in particular those aimed on the detailed mapping of the vorticity fields, are outlined. The Blast Wave model is used for an estimate of the anisotropic flow effect on the vorticity component along the beam direction. We also point to a possibility of a circular pattern in the vorticity field in asymmetric, e.g. $\mathrm{Cu}+\mathrm{Au}$, central collisions.
\end{abstract}

\section{Introduction}

The idea of the global polarization in heavy ion collisions, the phenomenon characterized by the polarization of the secondary particles along the global system orbital momentum, is almost 15 years old. It went "on-shell" in $2004[1,2]$ with the initial predictions for the hyperon polarization as high as "in the order of tens of a percent" [1]. The first measurements [3] by the STAR Collaboration of the lambda hyperon polarization in $\mathrm{Au}+\mathrm{Au}$ collisions at $200 \mathrm{GeV}$ put an upper limit on hyperon polarization of about $\left|P_{\Lambda}\right| \leq 0.02$. Subsequently, the theoretical predictions have been improved [4], especially with a better understanding of the statistical mechanics of vortical fluid with non-zero spin particles [5], and development of the hydrodynamical calculations assuming local angular momentum equilibrium. Rough estimate of the polarization can be obtained with the help of a simple nonrelativistic expression for a particle distribution in a fluid with nonzero vorticity [6] (for a strict relativistic consideration see [7]):

$$
p \propto \exp [-E / T-\omega(\mathbf{s}+\mathbf{l}) / T-\mu B / T],
$$

where $\omega=\frac{1}{2} \nabla \times \mathbf{v}$ is the nonrelativistic vorticity, and $\mathbf{v}$ is the fluid velocity. Figure 1 shows a cartoon of a non-central nuclear collision with arrows indicating the velocity field of the matter at the plane $z=0$. Just "guessing" that the difference in velocities in the "upper" and "lower" parts of the system is about a few tenths of the speed of light and that the transverse size of the system is about $10 \mathrm{fm}$, one would conclude that the vorticity might be at the level of a few percent of $\mathrm{fm}^{-1}$. Then the nonrelativistic formula (1) yields for the spin $1 / 2$ particle polarization, $P \approx \omega /(2 T)$, in the range of a few percent (assuming $T \sim 100 \mathrm{MeV}$ ).

\footnotetext{
^e-mail: sergei.voloshin@wayne.edu
} 


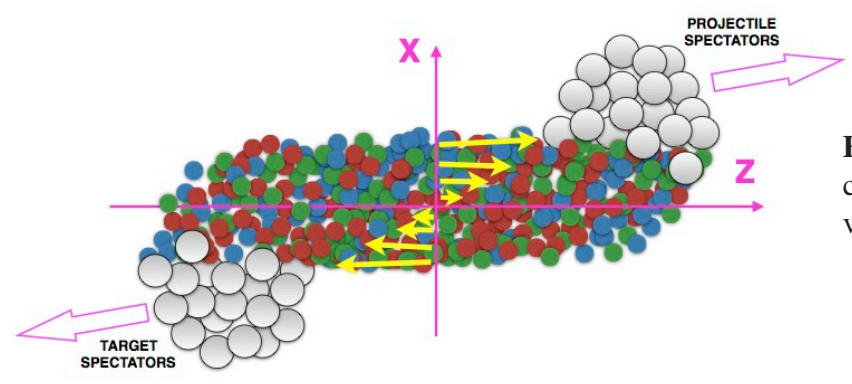

Figure 1. Cartoon of a non-central nuclear collision. The arrows indicate the collective velocity of the matter at $z=0$ plane.

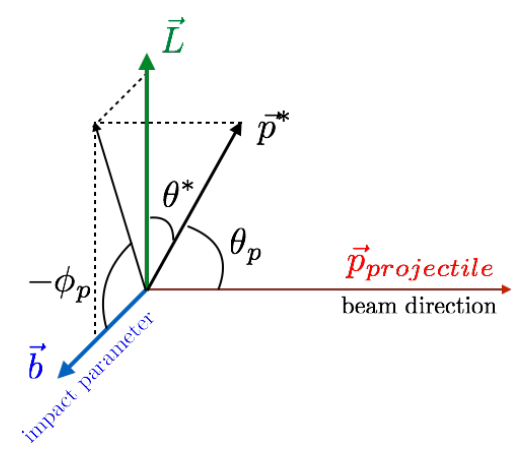

Figure 2. Diagram explaining the notations for different angles discussed in the text. $\mathbf{p}^{*}$ is the proton momentum in the hyperon rest frame. Vertical direction is the direction of the global orbital momentum - the global polarization direction. $\phi_{p}$ is the proton emission azimuth in the system with $x-z$ plane aligned with the reaction plane.

The simplest way to measure the global polarization is via analysis of the angular distributions of the products of weakly decaying hyperons. Weak interaction violates parity, and, e.g. in the lambda hyperon decay the protons are emitted preferentially in the direction of the lambda's spin:

$$
\frac{d N}{d \cos \theta^{*}} \propto 1+\alpha_{H} P_{H} \cos \theta^{*}
$$

where $\theta^{*}$ is the polar angle of the proton emission relative to the polarization direction in the hyperon rest frame, $-1 \leq P_{H} \leq 1$ is the hyperon polarization, and the parameter $\alpha_{\Lambda}=-\alpha_{\bar{\Lambda}} \approx 0.624$. To measure the polarization of strongly decaying particles is obviously significantly more difficult. It is not at all possible for spin 1/2 particles; for the vector mesons one can hope to measure the deviation from $1 / 3$ of the probability for the spin projection to be zero [8]. The angular distribution (averaged over the azimuthal distribution around the polarization direction) of the decay products in this case reads:

$$
\begin{aligned}
\frac{d N}{d \cos \theta^{*}} & \propto \rho_{0,0}\left|Y_{1,0}\right|^{2}+\rho_{1,1}\left|Y_{1,-1}\right|^{2}+\rho_{-1,-1}\left|Y_{1,1}\right|^{2} \propto \rho_{0,0} \cos ^{2} \theta^{*}+\frac{1}{2}\left(\rho_{1,1}+\rho_{-1,-1}\right) \sin ^{2} \theta^{*} \\
& \propto\left(1-\rho_{0,0}\right)+\left(3 \rho_{0,0}-1\right) \cos ^{2} \theta^{*}
\end{aligned}
$$

where $\rho_{0,0}, \rho_{1,1}$, and $\rho_{-1,-1}$ are the probabilities for the particle to have spin projection on the direction of polarization to be zero, +1 , and -1 , respectively. The deviation from the non-polarized state value $\rho_{0,0}=1 / 3$ is in this case a second order effect [8]. For example, an estimate based on Eq. 1 yields $\rho_{00}=1 /\left[3+(\omega / T)^{2}\right]$.

To measure the hyperon global polarization or vector meson spin alignment experimentally, one can either analyze directly the distributions in $\theta^{*}$, or, in case of the global polarization direction defined by the one of the flow event planes, analyze the azimuthal distribution of the decay products (in the resonance rest frame) relative to that flow plane. The azimuthal distribution analysis can be 
often simpler in terms of applying necessary corrections for the reaction plane resolution and detector acceptance. In the case of the azimuthal analysis, the polarization can be calculated as:

$$
\begin{aligned}
P_{H} & =-\frac{8}{\pi \alpha_{H}}\left\langle\sin \left(\phi_{P}^{*}-\Psi_{\mathrm{RP}}\right)\right\rangle, \\
\rho_{00} & =\frac{1}{3}-\frac{8}{3}\left\langle\cos \left[2\left(\phi_{p}^{*}-\Psi_{\mathrm{RP}}\right)\right]\right\rangle .
\end{aligned}
$$

For the global polarization, the analysis has to be performed using one of the first harmonic event planes, with the reaction plane (and correspondingly, the angular momentum) direction to be determined by the deflection direction of the projectile spectators (which on average deflect outward of the collision [9]). For the spin alignment measurements it is possible to use the second order event plane (which typically has much better resolution).

\section{Results}

The progress in vector spin alignments measurements was presented at this conference in talks by the STAR and ALICE Collaborations $[10,11]$. The uncertainties in these measurements are still relatively large, and the results are rather inconclusive; below I concentrate on the discussion of the global polarization results.

Figure 3 shows a compilation of published[3,12] and presented at this conference $[10,11]$ results on the average global polarization of lambda and anti-lambda hyperons at mid-rapidity in mid-central collisions as a function of collision energy. The blue solid and dashed lines are the results of hydrodynamic calculation [7, 13], with and without accounting for the hyperon feed-down contribution, respectively. The procedure for the feed-down correction is outlined in [6] with Eq. 1 used for an estimate of the polarization of the higher spin resonances. Note that the effect of the feed-down correction is rather modest - at the level of $\sim 15 \%$.

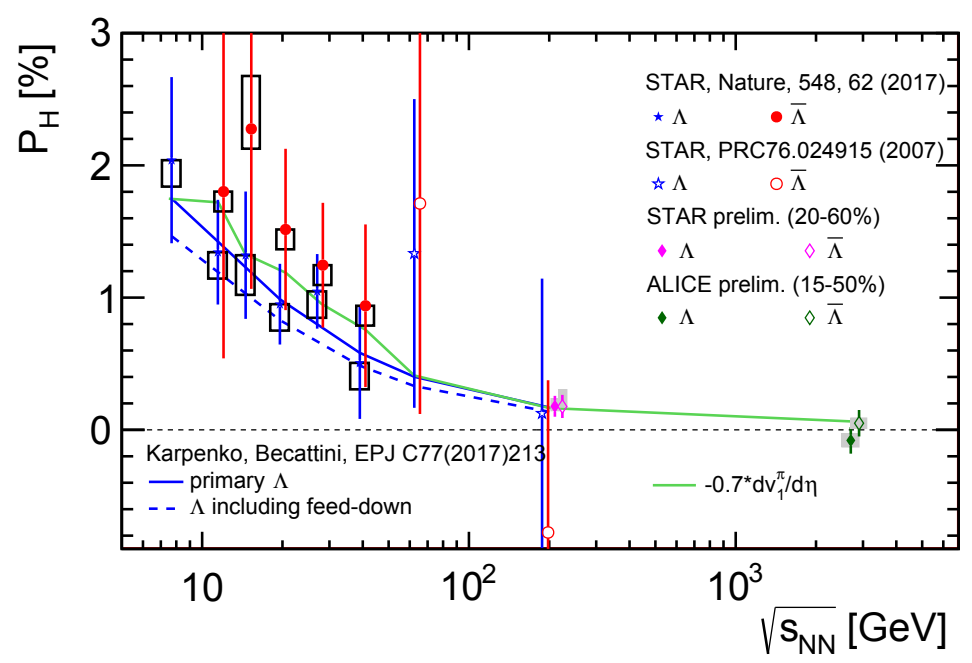

Figure 3. Average global polarization of lambda hyperons as a function of collision energy. Boxes indicate the systematic uncertainties. 
At RHIC beam energy scan collision energies the global polarization of lambdas and antilambdas seems to be slightly different. Calculations [14] show that the effect of nonzero baryon chemical potential could result in a split of the polarization values for particle and antiparticle, but numerically (assuming the equilibrium in the hadronic gas) this effect expected to be small. More realistic explanation for the difference would be the effect of the magnetic field that is strongly aligned with the direction of the orbital momentum. Then the global polarization analysis might become a very sensitive tool for the measurement of the magnetic field in heavy ion collisions. The present uncertainties in polarization measurements allow to put only an upper limit on the magnitude of the magnetic field, $e B \lesssim 0.01 m_{\pi}^{2}$, but even that is of great interest, as at present, the uncertainty in the magnetic field calculations spans several orders of magnitude [15].

There exist no hydrodynamic calculations for the global polarization at the LHC energies. For that we can develop an empirical prediction based on the observation that the slope of the directed flow at mid-rapidity is likely strongly correlated with the vorticity. Indeed, the calculation [16] support such a hypothesis. Then one can use the available data on $v_{1}$ slope $[17,18]$ (we used that of charged pions) for an estimate of the global polarization at higher energies. This is shown by a green line in Fig. 3. The ALICE global polarization measurements [11, 19], which within the error-bars at present are consistent with zero, do not contradict such an estimate. It will be required to improve the uncertainties about factor of 3-5 to be able to measure the polarization at the level of an estimate from the directed flow slopes.
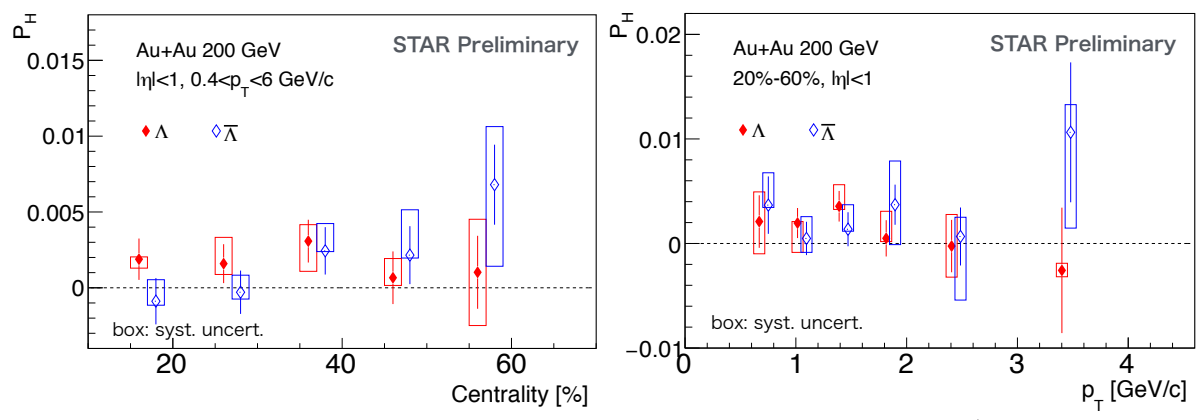

Figure 4. Centrality and $p_{T}$ dependence of the global polarization in $\mathrm{Au}+\mathrm{Au}$ collisions at $200 \mathrm{GeV}[10,20]$.

Global polarization dependence on centrality, the hyperon transverse momentum, and the orientation of the hyperon emission relative to the reaction plane have been also of great interest as different models predict significantly different dependencies. Within the available statistic, both, the centrality and $p_{\mathrm{T}}$ dependencies, see the preliminary STAR results $[10,20]$ presented in Fig. 4, are rather modest. The azimuthal angle dependence is discussed in more detail in [10]. The preliminary results indicate stronger polarization of the hyperons emitted in-plane than those emitted out-of-plane, opposite to the expectations from the hydrodynamical calculations [21].

\subsection{Global polarization and chiral anomalous effects}

The exact mechanism relating the vorticity of the medium to the global polarization is not yet firmly established. It was argued in [22] that the global polarization appears as a results of the axial anomalous current:

$$
\mathbf{J}_{5}=\left(\frac{\mu^{2}+\mu_{5}^{2}}{4 \pi^{2}}+\frac{T^{2}}{12}\right) \omega
$$


For a more detailed discussion of this possibility, see [23] and references therein.

Axial current can be also "generated" by the magnetic field in the system with nonzero vector charge chemical potential (for a review of chiral anomalous effects in heavy ion collisions see [24]):

$$
\mathbf{J}_{5}=\frac{1}{2 \pi^{2}} \mu_{V}(Q e) \mathbf{B},
$$

which in particular relates the direction of the axial current to the sign of the chemical potential. This provides an interesting possibility for the experimental check of the existence of such a relationship [25] by measuring the global polarization as a function of the event charge asymmetry or the net kaons, hoping that those are proportional to either electrical charge or strangeness chemical potentials. In some sense such a measurement would constitute a measurement of the first "half" of the chiral magnetic wave mechanism (in which the first step is the separation of the axial charge and the second step includes the electric currents rearranging the electric charge and finally leading to the quadrupole charge configuration, see review [24]). The preliminary results from the STAR Collaboration [20] presented in Fig. 5 are not yet conclusive due to the large statistical uncertainties.

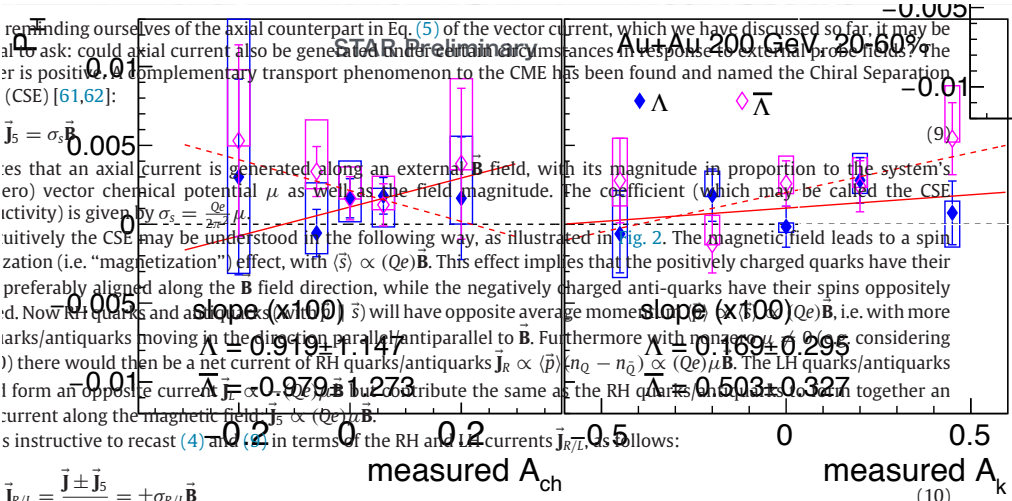

Figure 5. Global polarization as a function of the event charge asymmetry $A_{c h}=\left(N_{+}-N_{-}\right) /\left(N_{+}+N_{-}\right)$, and the event net kaon ratio $A_{K}=\left(N_{K^{+}}-N_{K^{-}}\right) /\left(N_{K_{+}}+N_{K^{-}}\right)[20]$.

\subsection{Local vorticity and polarization along the beam direction}

While the system orbital momentum provides an obvious choice for the average direction of the vorticity (and particle polarization), locally, the vorticity have in general nonzero all three components. Those might be generated by a particular process, e.g. jet propagation [26] (not yet studied experimentally) or simply by the expansion of the initially anisotropic medium, see, for example, the results in [16] (including those presented only in the arXiv version of this publication), as well as calculations in $[14,22]$. In particular, the vorticity $z$-component, along the beam direction, might reach large values $[7,13,16]$.

Below I discuss a simple Blast Wave (BW) model estimates for the vorticity $z$ component. In a simplest version of BW model that includes anisotropic flow, the particle production source at freezeout is parameterized with 4 parameters: temperature $T$, maximum radial flow velocity (rapidity), 


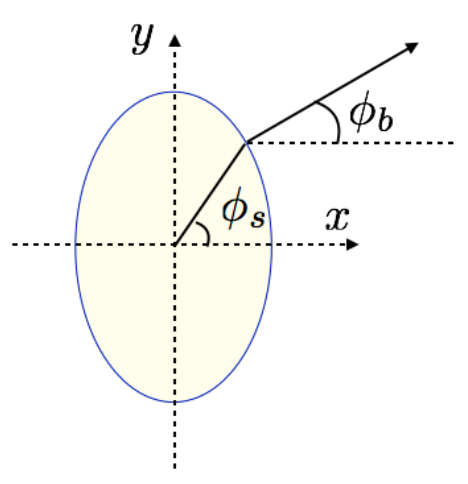

Figure 6. The collective velocity of the source element at angle $\phi_{s}$ at the surface is along the boost angle $\phi_{b}$, perpendicular to the surface described by Eq. 9. The boost velocity is given by Eq. 10 .

notated as $\rho_{0}$, amplitude of azimuthal modulation in expansion velocity, noted below as $b$, and the spatial anisotropy parameter $a$. The source (see Fig. 6) is then described by the following equations:

$$
\begin{gathered}
r_{\text {max }}=R\left[1-a \cos \left(2 \phi_{s}\right)\right], \\
\rho_{t}=\rho_{t, \text { max }}\left[r / r_{\text {max }}\left(\phi_{s}\right)\right]\left[1+b \cos \left(2 \phi_{s}\right)\right] \approx \rho_{t, \text { max }}(r / R)\left[1+(a+b) \cos \left(2 \phi_{s}\right)\right] .
\end{gathered}
$$

It is assumed that the collective velocity of the source element located at azimuthal angle $\phi_{s}$ is boosted with velocity $\rho_{t}$ perpendicular to the surface of the ellipse similar to that of Eq. 9. Assuming that $a \ll 1, b \ll 1$, the difference $\phi_{s}-\phi_{b} \approx 2 a \sin \left(2 \phi_{s}\right)$ and the vorticity:

$$
\omega_{z}=1 / 2(\nabla \times \mathbf{v})_{z} \approx\left(\rho_{t, n \max } / R\right) \sin \left(n \phi_{s}\right)\left[b_{n}-a_{n}\right] .
$$

The estimates above should be valid for anisotropic flow of any harmonics - which is the reason we have changed in Eq. 11 the harmonic order from 2 to $n$. It is obviously quite a rough approximation (which in principle can be improved) as it leads to a discontinuity at the origin. It provides the following estimate for the hyperon polarization:

$$
P_{z} \approx \omega_{z} /(2 T) \approx 0.1 \sin \left(n \phi_{s}\right)\left[b_{n}-a_{n}\right],
$$

where we assumed that $\rho_{t, n \max } \sim 1, R \approx 10 \mathrm{fm}$, and $T \approx 100 \mathrm{MeV}$. In practice, the coefficients $b_{n}$ and $a_{n}$ are both of the order of a few percent, often close to each other. That results in the values for $z$-polarization not greater than a few per-mill, almost an order of magnitude lower than obtained in hydrodynamics calculations [7, 13].

The measurements of the $z$ component of polarization could be relatively simple as they do not require the knowledge of the first harmonic event plane. The acceptance effects should be also readily accounted for requiring that the $z$ component of the polarization averaged over all azimuthal angles to be zero.

We note that vorticity fields due the anisotropic flow are formed closer to the freeze-out, unlike the ones due to the "shear" in the initial velocity fields (as shown in Fig 1). Having in mind the finite relaxation time for establishing the equilibrium the relation between these two vorticities and the final polarizations can be different.

Finally, we mention another very interesting possibility for vorticity studies in asymmetric nuclear collisions such as $\mathrm{Cu}+\mathrm{Au}$. For relatively central collisions, when during the collision a smaller nucleus is fully "absorbed" by the larger one (e.g. such collisions can be selected by requiring no signal in the zero degree calorimeter in the lighter nucleus beam direction), one can easily imagine a configuration with toroidal velocity field, and as a consequence, a vorticity field in the form of a circle. The direction of the polarization in such a case would be given by $\hat{\mathbf{p}_{\mathrm{T}}} \times \hat{\mathbf{z}}$, where $\hat{\mathbf{p}_{\mathrm{T}}}$ and $\hat{\mathbf{z}}$ are the unit vectors along the particle transverse momentum and the (lighter nucleus) beam direction. 


\section{Summary}

The hyperon polarization measurements provide a unique possibility to study the velocity fields in heavy ion collisions. The difference in particle//antiparticle polarization might provide a tool for the magnetic field measurements. Studing the global polarization as a function of event net electrical charge or strangeness could establish the relation between the global polarization and the chiral anomalous effect. The future $28 \mathrm{GeV} \mathrm{Au+Au} \mathrm{RHIC} \mathrm{run,} \mathrm{as} \mathrm{well} \mathrm{as} \mathrm{the} \mathrm{future} \mathrm{LHC} \mathrm{runs,} \mathrm{will} \mathrm{bring} \mathrm{a}$ high statistics data sets suitable for many differential studies outlined in this presentation.

Very fruitful discussions with F. Becattini, Yu. Karpenko, M. Konyushikhin, M. Lisa, T. Niida, I. Upsal, and X.-N. Wang are greatly appreciated. This material is based upon work supported in part by the U.S. Department of Energy Office of Science, Office of Nuclear Physics under Award Number DE-FG02-92ER-40713.

\section{References}

[1] Z. T. Liang and X. N. Wang, "Globally polarized quark-gluon plasma in non-central A+A collisions," Phys. Rev. Lett. 94, 102301 (2005) Erratum: [Phys. Rev. Lett. 96, 039901 (2006)] doi:10.1103/PhysRevLett.94.102301 [nucl-th/0410079].

[2] S. A. Voloshin, "Polarized secondary particles in unpolarized high energy hadron-hadron collisions?," nucl-th/0410089.

[3] B. I. Abelev et al. [STAR Collaboration], "Global polarization measurement in $\mathrm{Au}+\mathrm{Au}$ collisions," Phys. Rev. C 76, 024915 (2007) doi:10.1103/PhysRevC.76.024915

[4] J. H. Gao, S. W. Chen, W. t. Deng, Z. T. Liang, Q. Wang and X. N. Wang, "Global quark polarization in non-central A+A collisions," Phys. Rev. C 77, 044902 (2008) doi:10.1103/PhysRevC.77.044902 [arXiv:0710.2943 [nucl-th]].

[5] F. Becattini and F. Piccinini, "The Ideal relativistic spinning gas: Polarization and spectra," Annals Phys. 323, 2452 (2008) doi:10.1016/j.aop.2008.01.001

[6] F. Becattini, I. Karpenko, M. Lisa, I. Upsal and S. Voloshin, "Global hyperon polarization at local thermodynamic equilibrium with vorticity, magnetic field and feed-down," Phys. Rev. C 95, no. 5, 054902 (2017) doi:10.1103/PhysRevC.95.054902

[7] F. Becattini, these proceedings

[8] Z. T. Liang and X. N. Wang, "Spin alignment of vector mesons in non-central A+A collisions," Phys. Lett. B 629, 20 (2005) doi:10.1016/j.physletb.2005.09.060

[9] S. A. Voloshin and T. Niida, "Ultrarelativistic nuclear collisions: Direction of spectator flow," Phys. Rev. C 94, no. 2, 021901 (2016) doi:10.1103/PhysRevC.94.021901

[10] Biao Tu, for the STAR Collaboration, these proceedings

[11] B. Mohanty, for the ALICE Collaboration, these proceedings

[12] L. Adamczyk et al. [STAR Collaboration], "Global $\Lambda$ hyperon polarization in nuclear collisions: evidence for the most vortical fluid,” Nature 548, 62 (2017) doi:10.1038/nature23004

[13] Yu. Karpenko, these proceedings

[14] R. h. Fang, L. g. Pang, Q. Wang and X. n. Wang, "Polarization of massive fermions in a vortical fluid," Phys. Rev. C 94, no. 2, 024904 (2016) doi:10.1103/PhysRevC.94.024904

[15] L. McLerran and V. Skokov, "Comments About the Electromagnetic Field in Heavy-Ion Collisions," Nucl. Phys. A 929, 184 (2014) doi:10.1016/j.nuclphysa.2014.05.008

[16] F. Becattini et al., "A study of vorticity formation in high energy nuclear collisions," Eur. Phys. J. C 75, no. 9, 406 (2015) doi:10.1140/epjc/s10052-015-3624-1 
[17] S. Singha, P. Shanmuganathan and D. Keane, "The first moment of azimuthal anisotropy in nuclear collisions from AGS to LHC energies," Adv. High Energy Phys. 2016, 2836989 (2016) doi: $10.1155 / 2016 / 2836989$

[18] B. Abelev et al. [ALICE Collaboration], "Directed Flow of Charged Particles at Midrapidity Relative to the Spectator Plane in Pb-Pb Collisions at $\sqrt{s_{N N}}=2.76 \mathrm{TeV}$," Phys. Rev. Lett. 111, no. 23, 232302 (2013) doi:10.1103/PhysRevLett.111.232302

[19] M. Konyushikhin for the ALICE Collaboration, poster at Quark Matter 2017 conference

[20] T. Niida for the STAR Collaboration, talk at QCD Chirality workshop, UCLA, 2017.

[21] F. Becattini, L. Csernai and D. J. Wang, " $\Lambda$ polarization in peripheral heavy ion collisions," Phys. Rev. C 88, no. 3, 034905 (2013) Erratum: [Phys. Rev. C 93, no. 6, 069901 (2016)] doi:10.1103/PhysRevC.93.069901, 10.1103/PhysRevC.88.034905

[22] M. I. Baznat, K. K. Gudima, A. S. Sorin and O. V. Teryaev, "Femto-vortex sheets and hyperon polarization in heavy-ion collisions," Phys. Rev. C 93, no. 3, 031902 (2016) doi:10.1103/PhysRevC.93.031902

[23] O. Teryaev, these proceedings

[24] D. E. Kharzeev, J. Liao, S. A. Voloshin and G. Wang, "Chiral magnetic and vortical effects in high-energy nuclear collisions-A status report," Prog. Part. Nucl. Phys. 88, 1 (2016) doi:10.1016/j.ppnp.2016.01.001

[25] S. Schlihting and S. Voloshin, in preparation

[26] B. Betz, M. Gyulassy and G. Torrieri, "Polarization probes of vorticity in heavy ion collisions," Phys. Rev. C 76, 044901 (2007) doi:10.1103/PhysRevC.76.044901 